\title{
Sub-nm Spectroscopic Mapping of Highly Beam Sensitive Species Using Direct Detection Electron Energy Loss Spectroscopy
}

\author{
M. Cem Akatay ${ }^{1}$ \\ 1. UOP LLC, A Honeywell Company, Des Plaines, Illinois, USA. \\ *cem.akatay@honeywell.com
}

Nanoscale spectroscopic mapping is an integral part of nanomaterials research and development. Aberration corrected transmission electron microscopes are able to focus the electron beam to a subAngstrom probe paving the way for sub-nanometer analysis, but spectroscopic mapping of the desired elements within the same degree of magnification often leads to structural damage caused by the high electron dose of the finely focused probe [1]. Direct electron detectors (DED) have been adapted for electron energy loss spectroscopy in place of the conventional indirect detectors that are based on charged coupled device (CCD) cameras and enable low dose spectroscopic mapping at nanoscale [2]. Direct electron detectors count single electrons and eliminate the step of photon conversion resulting in improved detective quantum efficiency (DQE) coupled with superior signal-to-noise ratio (SNR). Combining these characteristics with faster readout speed, the DED-EELS is a powerful tool for mapping beam sensitive species at the nanoscale $[3,4]$.

In the present study, the quality of $\mathrm{Na}(\mathrm{Z}=11)$ distribution on the $\mathrm{Al}_{2} \mathrm{O}_{3}$ was studied. $\mathrm{Na}$ was used to modify the alumina and uniform distribution of the $\mathrm{Na}$ is required for better performance. The $\mathrm{Na}$ loading was determined to be around $15 \mathrm{wt} \%$. By means of imaging, it is impossible to distinguish $\mathrm{Na}$ species from the underlying alumina. In order to assess the $\mathrm{Na}$ distribution at the sub-nanometer scale, chemical mapping of the material was done using three different configurations available on the aberration corrected FEI Titan 80-300kV transmission electron microscope at the operating extraction voltage of $300 \mathrm{kV}$. Energy dispersive X-ray (EDX) spectroscopy data was collected by using ChemiSTEM $^{\mathrm{TM}}$ (4 SDD detectors in the Super-X ${ }^{\mathrm{TM}}$ geometry). The electron energy loss spectroscopy was performed using Gatan's Quantum ${ }^{\circledR}$ Imaging Filter either using the conventional CCD or the direct electron detector (K2).

$\mathrm{Na}$ and $\mathrm{Al}$ EDX maps were acquired with $1 \mu$ s dwell time using KA line for $\mathrm{Na}(\mathrm{E}=1.040 \mathrm{keV})$ and $\mathrm{K}$ line for $\mathrm{Al}(\mathrm{E}=1.487 \mathrm{keV})$. The maps showed uniform $\mathrm{Na}$ distribution on the alumina for acquisitions where the pixel size was larger than $1 \mathrm{~nm}$. For acquisitions with sub-nanometer pixel size, the $\mathrm{Na}$ species were mobilized by the electron beam resulting in the displacement of Na species out of the alumina (Figure 1A). CCD-EELS maps were collected with $50 \mathrm{~ms}$ dwell time at the Na K edge employing an electron dose 5 times lower than the one used for EDX. Again, the electron beam has swept the Na species to the side (Figure 1B). Lastly, DED-EELS was used at an electron dose 10 times lower than the one used for CCD-EELS and with the dwell time of $2.5 \mathrm{~ms} /$ pixel. This time, Na was mapped without being mobilized for sub-nanometer pixel size and the resulting maps showed uniform $\mathrm{Na}$ distribution over the alumina (Figure 1C).

This study was one of the many examples where DED-EELS makes a difference in the characterization of industrially relevant materials. Additional examples will be discussed including DED-EELS characterization of carbonaceous species on beam-sensitive industrial zeolites. 


\section{References:}

[1] JC Meyer, J. Kotakoski, C. Mangler, Ultramicroscopy 145 (2014) 13

[2] JA Hart et al, Scientific Reports 7 (2017) 8243

[3] RS Ruskin, Z Yu, and N Grigorieff, J. Struct. Biol. 184 (2013), p. 385

[4] JL Hart, AC Lang, C Trevor, R Twesten, ML Taheri, Microsc. Microanal. 22 (S3) (2016), p. 336
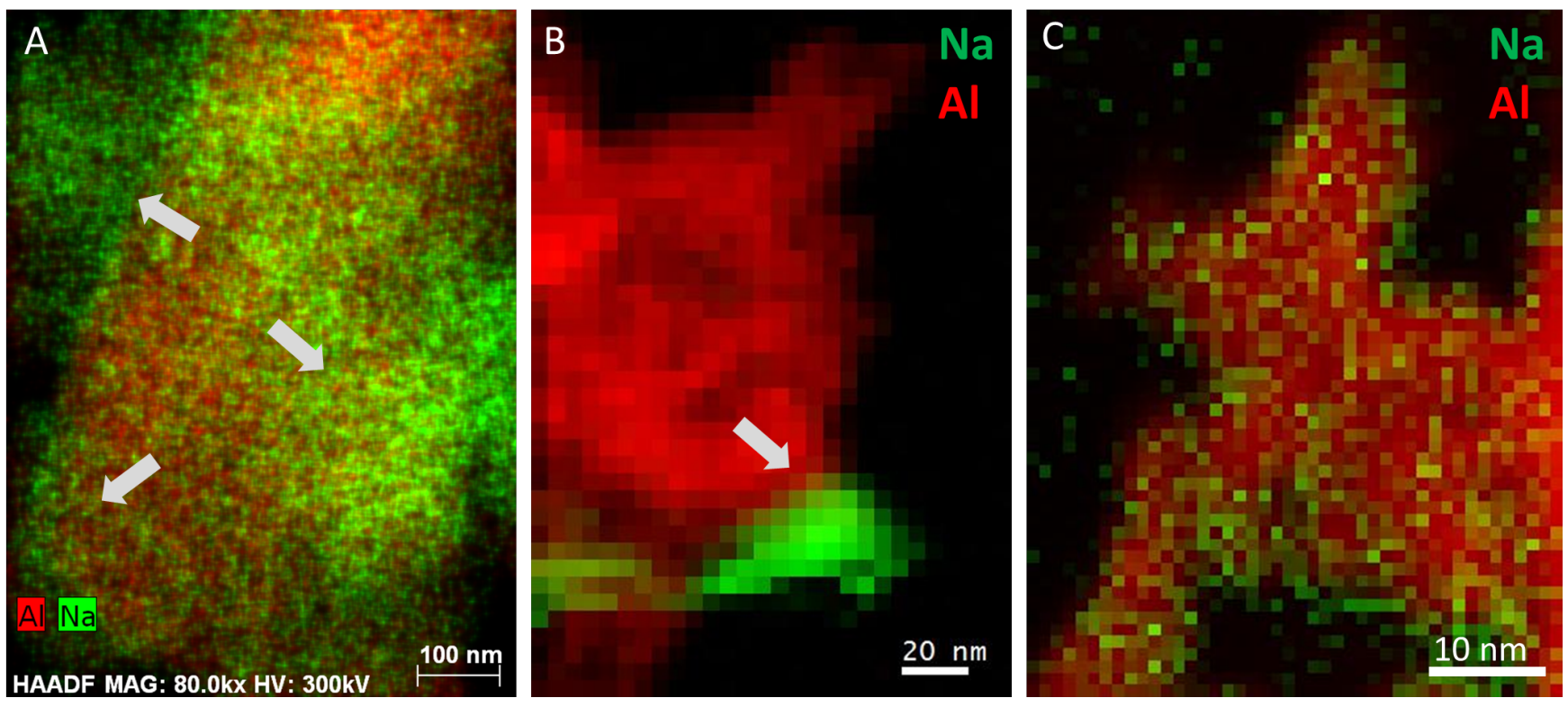

Figure 1. $\mathrm{Al} \mathrm{K}$ and $\mathrm{Na} \mathrm{K}$ edge elemental maps of 15 wt. $\% \mathrm{Na} / \mathrm{Al}_{2} \mathrm{O}_{3}$. Acquired by (A) ChemiSTEM ${ }^{\mathrm{TM}}$ EDX (B) CCD coupled EELS (C) Direct Detection EELS. Mobilization of Na under electron beam is evident on (A) and (B), whereas on (C) Na remained unperturbed despite higher resolution mapping 